\title{
Low Reynolds Number Swimming with Slip Boundary Conditions
}

\author{
Hashim Alshehri, Nesreen Althobaiti, and Jian Du ${ }^{(凶)}$ \\ Florida Institute of Technology, Melbourne, FL 32901, USA \\ jdu@fit.edu
}

\begin{abstract}
We investigate the classical Taylor's swimming sheet problem in a viscoelastic fluid, as well as in a mixture of a viscous fluid and a viscoelastic fluid. Extensions of the standard Immersed Boundary (IB) Method are proposed so that the fluid media may satisfy partial slip or free-slip conditions on the moving boundary. Our numerical results indicate that slip may lead to substantial speed enhancement for swimmers in a viscoelastic fluid and in a viscoelastic two-fluid mixture. Under the slip conditions, the speed of locomotion is dependent in a nontrivial way on both the viscosity and elasticity of the fluid media. In a two-fluid mixture with free-slip network, the swimming speed is also significantly affected by the drag coefficient and the network volume fraction.
\end{abstract}

Keywords: Swimming sheet $\cdot$ Viscoelastic fluid $\cdot$ Slip condition · Immersed boundary method

\section{Introduction}

How micro-organisms move in their surrounding fluid environment is of significant biological and clinical importance. Examples include the locomotion of E.coli in intestinal fluid [1], and the swimming of mammalian spermatozoa within cervical mucus in the process of reproduction [2]. Such problems involve the dynamical interactions between elastic boundaries and a complex fluid medium, which often exhibits complicated Non-Newtonian responses. Recent theoretical, experimental and computational investigations are characterized by the complexity of different ways in which biological locomotion may depend on fluid properties. Analysis of the infinite undulatory sheet with small amplitude found that fluid elasticity always reduces the swimming speed [3]. Further analytical work indicated that swimming can be boosted by elasticity under specified gaits [4]. Numerical simulations of finite swimmers with large amplitude of motion showed that swimming speed may be enhanced by elasticity [5]. Experimentally, the self-propulsion of C. elegans was observed to be hindered significantly in viscoelastic fluid [6]. However, the artificial swimmers in [7] exhibited systematic elastic speed-ups. In [8] and [9], it was shown that favorable stroke asymmetry, swimmer body dynamics and fluid elasticity may work together to cause increases in speed.

(C) Springer Nature Switzerland AG 2020

V. V. Krzhizhanovskaya et al. (Eds.): ICCS 2020, LNCS 12141, pp. 149-162, 2020.

https://doi.org/10.1007/978-3-030-50426-7_12 
In most of the analytical and numerical works to date, the fluid environment is treated as a single continuous medium. No-slip boundary condition is assumed on the swimmer's surface so that the fluid medium always moves together with the swimmer. Such models and assumptions may not be appropriate for many applications. First, biological fluids such as mucus are mixtures of a solvent and a polymer network. There may be significant relative motions between different components within the mixture so that it can not be adequately described by a single phase continuum medium [10]. Furthermore, it has been long known that slip may occur for polymer solutions near a solid boundary. This can be caused by the phase separation over the solvent-rich boundary region where the polymer phase is driven away [11]. Recent studies highlight the importance of boundary conditions and fluid models in locomotion problems. The analysis in [12] examined swimming in a medium consisting of a mixture of a Newtonian fluid and an elastic solid. Both elastic speed-up and slow-down can be obtained, depending on the type of boundary conditions imposed. In [13], it was shown analytically that the introduction of apparent slip or the reduction of fluid viscosity near the swimmer in Newtonian fluids may lead to faster swimming. In [14] and [15], different variations of the Immersed Boundary Method were proposed to simulate interactions between elastic boundaries and a two-phase medium. Despite these advances, a comprehensive analysis for the role of slip on swimmers in viscoelastic media is lacking.

In this paper, we present the first computational investigation of the role of slip for Taylor's classical swimming sheet in a single phase viscoelastic fluid, as well as in a mixture of a viscous fluid and a viscoelastic fluid. Our computational method is based on extensions of the classical Immersed Boundary Method [16] so that elastic boundaries are allowed to slip through the surrounding fluid media. In Sect. 2 and 3, the model equations and numerical methods are presented first, followed by simulation results which highlight the influence of slip on locomotion in complex fluids. The concluding remarks are given in Sect. 4.

\section{Swimming in a Single Phase Viscous/Viscoelastic Fluid}

\section{$2.1 \quad$ Model Equations}

Consider an infinite 2D sheet immersed in a incompressible, viscoelastic OldroydB fluid. In its own frame, the movement of the sheet is described by $\mathrm{y}=\epsilon \sin (\mathrm{kx}-$ $\omega t)$. The fluid equations are given by:

$$
\begin{gathered}
\nabla \cdot \boldsymbol{\sigma}-\nabla \mathrm{p}=0, \\
\nabla \cdot \mathbf{u}=0,
\end{gathered}
$$

where $\mathbf{u}$ is the fluid velocity, and $\mathrm{p}$ is the pressure. The total stress tensor is composed of viscous and polymeric contributions: $\boldsymbol{\sigma}=\mu_{\mathrm{s}}\left(\nabla \mathbf{u}+\nabla \mathbf{u}^{\mathrm{T}}\right)+\boldsymbol{\sigma}_{\mathrm{p}}$, with 
$\mu_{\mathrm{s}}$ be the shear viscosity of the fluid. The polymer stress $\boldsymbol{\sigma}_{\mathrm{p}}$ evolves according to constitutive equation:

$$
\boldsymbol{\sigma}_{\mathrm{p}}+\lambda\left(\frac{\partial \boldsymbol{\sigma}_{\mathrm{p}}}{\partial \mathrm{t}}+\mathbf{u} \cdot \nabla \boldsymbol{\sigma}_{\mathrm{p}}-\nabla \mathbf{u}^{\mathrm{T}} \cdot \boldsymbol{\sigma}_{\mathrm{p}}-\boldsymbol{\sigma}_{\mathrm{p}} \cdot \nabla \mathbf{u}\right)=\mu_{\mathrm{p}}\left(\nabla \mathbf{u}+\nabla \mathbf{u}^{\mathrm{T}}\right) .
$$

Here $\mu_{\mathrm{p}}$ is the polymer viscosity and $\lambda$ is the polymer relaxation time. On the sheet surface $\Gamma$, the fluid velocity $\mathbf{u}$ satisfies the following boundary conditions:

$$
\begin{gathered}
{\left.[\mathbf{u} \cdot \mathbf{n}]\right|_{\Gamma}=0 .} \\
{\left.[\mathbf{u} \cdot \boldsymbol{\tau}]\right|_{\Gamma}=\left.2 \Xi(\boldsymbol{\tau} \cdot \boldsymbol{\sigma} \cdot \mathbf{n})\right|_{\Gamma} .}
\end{gathered}
$$

$\mathbf{n}$ and $\boldsymbol{\tau}$ are unit vectors normal and tangential to the surface, respectively. The square bracket terms represent the components of the fluid velocity relative to the surface of the sheet (slip velocity). $\Xi$ is the slip coefficient. Condition (4) states that the fluid and the sheet move together in the direction normal to the sheet surface. According to (5), the fluid is allowed to slip relative to the sheet in its tangential direction. The extent of slip is proportional to the local shear stress, as well as the slip constant $\Xi$. This is the well known Navier Slip Condition [17]. Note that the boundary conditions (4) and (5) apply to both the upper and lower surfaces of the sheet. Since Taylor's classical work [18], there have been many analytical and computational studies on different versions of the swimming sheet problem. See [19] for a complete review.

\subsection{IB Method with Partial Slip Condition}

The "classical" Immersed Boundary (IB) Method [16] is a powerful computational method capable of handling dynamic fluid-structure interactions. An Eulerian description is used for the fluid variables such as velocity and pressure, while a Lagrangian coordinate is used for each immersed elastic object. The simplicity and robustness of the IB method have led to its successful applications to many biological problems. Let $\mathbf{x}$ denote the fixed Eulerian coordinates and $\mathbf{X}(\mathrm{q}, \mathrm{t})$ be the physical location of material points on the immersed object, which is parameterized by q. Let $\Omega$ be the fluid domain and $\Gamma$ denote the Lagrangian domain. The equations for the coupled fluid-structure system are given by:

$$
\begin{gathered}
\nabla \cdot \boldsymbol{\sigma}-\nabla \mathrm{p}+\mathbf{f}=0 \\
\mathbf{f}(\mathbf{x}, \mathrm{t})=\int_{\Gamma} \mathbf{F}(\mathrm{q}, \mathrm{t}) \delta(\mathbf{x}-\mathbf{X}(\mathrm{q}, \mathrm{t})) \mathrm{dq}=\mathrm{S} \mathbf{F} \\
\frac{\partial \mathbf{X}(\mathrm{q}, \mathrm{t})}{\partial \mathrm{t}}=\int_{\Omega} \mathbf{u}(\mathbf{x}, \mathrm{t}) \delta(\mathbf{x}-\mathbf{X}(\mathrm{q}, \mathrm{t})) \mathrm{d} \mathbf{x}=\mathrm{S}^{*} \mathbf{u} .
\end{gathered}
$$

Here $\delta$ denotes the Dirac delta function. (7) describes how the Lagrangian force density $\mathbf{F}$ is spread to the fluid and $\mathrm{S}$ represents the force spreading operator. (8) is based on the assumption that the immersed object moves with local fluid 
velocity (no-slip condition). $\mathrm{S}^{*}$ is the velocity interpolation operator which is the adjoint of the spreading operator $\mathrm{S}$.

IB method described above needs to be modified to handle slip conditions such as (5). This involves the evaluation of the interfacial fluid stresses on the irregular boundary, which can be computationally challenging [20]. On a Stokes swimmer, the elastic force $\mathbf{F}$ is balanced by the hydrodynamics forces (both viscous and viscoelastic), which can be calculated from the jump in fluid stress across the swimmer. For Taylor's sheet within an infinite domain, the tangential hydrodynamics forces on the two surfaces $\left(\Gamma_{+}\right.$and $\left.\Gamma_{-}\right)$of the sheet are equal because of symmetry. So we have $\boldsymbol{\tau} \cdot \boldsymbol{\sigma}_{+} \cdot \mathbf{n}=-\boldsymbol{\tau} \cdot \boldsymbol{\sigma}_{-} \cdot \mathbf{n}$. Thus the force balance on the sheet in the tangential direction gives $\mathbf{F} \cdot \boldsymbol{\tau}=-\boldsymbol{\tau} \cdot[\boldsymbol{\sigma}] \cdot \mathbf{n}=-2 \boldsymbol{\tau}$. $\boldsymbol{\sigma}_{+} \cdot \mathbf{n}$, where $[\boldsymbol{\sigma}]=\boldsymbol{\sigma}_{+}-\boldsymbol{\sigma}_{-}$is the stress jump across the sheet. Therefore, the tangential component of the elastic force (which is straightforward to compute in IB method) can be directly used to enforce the slip boundary condition. Denote the boundary fluid velocity obtained from right hand side of (8) by $\mathbf{U}(\mathbf{X}(\mathrm{q}, \mathrm{t}))$, the sheet velocity $\mathbf{U}_{\Gamma}$ can then be computed by:

$$
\begin{gathered}
\mathbf{U}_{\Gamma}(\mathbf{X}) \cdot \mathbf{n}=\mathbf{U}(\mathbf{X}) \cdot \mathbf{n} \\
\mathbf{U}_{\Gamma}(\mathbf{X}) \cdot \boldsymbol{\tau}=\mathbf{U}(\mathbf{X}) \cdot \boldsymbol{\tau}+\Xi \mathbf{F} \cdot \boldsymbol{\tau} .
\end{gathered}
$$

\subsection{Discretization and Numerical Solutions}

All fluid variables are discretized using a Cartesian grid, with constant grid space h. A MAC-type staggered computational grid is used for spatial discretization. Scalars are located at the grid centers and vectors are located at the grid edges. All components of the viscoelastic stress tensor $\boldsymbol{\sigma}_{\mathrm{p}}$ are placed at the cell centers. The sheet is represented by a set of discrete IB points. Using centered difference for all spatial derivatives, the discretized equations from time $\mathrm{t}^{\mathrm{k}}$ to $\mathrm{t}^{\mathrm{k}+1}=\mathrm{t}^{\mathrm{k}}+\Delta \mathrm{t}$ are:

$$
\begin{gathered}
\mu_{\mathrm{s}} \Delta_{\mathrm{h}} \mathbf{u}^{\mathrm{k}+1}+\nabla_{\mathrm{h}} \cdot \boldsymbol{\sigma}_{\mathrm{p}}^{\mathrm{k}+1}-\nabla_{\mathrm{h}} \mathrm{p}^{\mathrm{k}+1}+S_{\mathrm{h}}^{\mathrm{k}} \mathbf{F}\left(\mathbf{X}^{\mathrm{k}}\right)=0, \\
\nabla_{\mathrm{h}} \cdot \mathbf{u}^{\mathrm{k}+1}=0, \\
\mathbf{X}^{\mathrm{k}+1}=\mathbf{X}^{\mathrm{k}}+\Delta \mathrm{t}\left(\left(S_{\mathrm{h}}^{*}\right)^{\mathrm{k}} \mathbf{u}^{\mathrm{k}+1}+\Xi\left(\mathbf{F}\left(\mathbf{X}^{\mathrm{k}}\right) \cdot \boldsymbol{\tau}^{\mathrm{k}}\right) \boldsymbol{\tau}^{\mathrm{k}}\right) .
\end{gathered}
$$

Here $\Delta_{\mathrm{h}}$ and $\nabla_{\mathrm{h}}$ are discretized Laplacian and gradient operators, respectively. $S_{\mathrm{h}}^{\mathrm{k}}$ and $S_{\mathrm{h}}^{*}$ are discretized version of the spreading and interpolation operators as defined in (7) and (8). The time iteration for the proposed scheme can be summarized as following:

1. Compute the elastic forces $\mathbf{F}\left(\mathbf{X}^{\mathrm{k}}\right)$ on the sheet from its geometric configuration at $\mathrm{t}^{\mathrm{k}}$. Spread the Lagrangian force to the fluid grid.

2. Update the viscoelastic stress tensor $\boldsymbol{\sigma}_{\mathrm{p}}^{\mathrm{k}+1}$ from the discretization of (3) using extrapolated velocity at time level $\mathrm{t}^{\mathrm{k}+1 / 2}$ from values at $\mathrm{t}^{\mathrm{k}}$ and $\mathrm{t}^{\mathrm{k}-1}$.

3. Solve (11) and (12) to get the values of $\mathbf{u}$ and $\mathrm{p}$ at $\mathrm{t}^{\mathrm{k}+1}$.

4. Update the positions of the IB points on the sheet according to (13). 
Each IB point is connected by linear springs to its two neighboring points. It is also connected by a stiff spring to a corresponding "tether" point whose role is to impose the desired motion of the sheet. The unit tangent vector $\tau_{\mathrm{j}}$ at the $\mathrm{j}^{\text {th }}$ IB point $\mathbf{X}_{\mathrm{j}}$ is approximated by $\boldsymbol{\tau}_{\mathrm{j}}=\frac{\boldsymbol{\tau}_{\mathrm{j}+1 / 2}+\boldsymbol{\tau}_{\mathrm{j}-1 / 2}}{2}$, where $\boldsymbol{\tau}_{\mathrm{j}+1 / 2}=\frac{\mathbf{X}_{\mathrm{j}+1}-\mathbf{X}_{\mathrm{j}}}{\left\|\mathbf{X}_{\mathrm{j}+1}-\mathbf{X}_{\mathrm{j}}\right\|}$. Surface normal $\mathbf{n}_{\mathrm{j}}$ is obtained by a $\pi / 2$ rotation of $\boldsymbol{\tau}_{\mathrm{j}}$. The discretized operators $S_{\mathrm{h}}^{\mathrm{k}}$ and $S_{\mathrm{h}}^{*}$ are constructed with the four-point cosine-based discrete delta function proposed by Peskin [16]. A multigrid solver with the box-type smoother is used to solve the coupled linear system from (11) and (12) [21]. Finally, to solve the stress Eq. (3), a high-resolution unsplit Godunov scheme is used to approximate the advection term explicitly. Crank-Nicolson approximation is used for the remaining terms. For each Eulerian grid cell, a $3 \times 3$ linear system is solved to update all components of $\boldsymbol{\sigma}_{\mathrm{p}}$. See [22] for the detailed algorithm.

Our simulations are carried out in the domain $[0,1] \times[-1,1]$. The boundary condition in the $\mathrm{x}$ direction is periodic and that at $y= \pm 2$ is no-slip. The grid size is $128 \times 256$ and a constant time step $\Delta t=10^{-4}$ is used for all simulations. For all results presented in this paper, we use $\epsilon=0.012, \mathrm{k}=\omega=2 \pi$, and $\mu_{\mathrm{s}}=1$. The swimming speed of the sheet is calculated by averaging the $\mathrm{x}$ velocity over all the IB points and over one wave period until a steady state value is obtained. To verify the proposed method, we first set $\boldsymbol{\sigma}_{\mathrm{p}}$ to zero and compare the numerical results with the analytical solution given by [13]:

$$
\frac{\mathrm{U}}{\mathrm{U}_{0}}=1+4 \mathrm{k} \mu_{\mathrm{s}} \Xi
$$

where $\mathrm{U}$ and $\mathrm{U}_{0}$ are the second order swimming speeds of the sheet with and without slip, respectively. The no-slip swimming speed is given by $\mathrm{U}_{0}=-\frac{1}{2} \mathrm{k} \omega \epsilon^{2}$. Note that the slip velocity in [13] is proportional to the shear rate, instead of the shear stress. So the slip length $\Lambda$ as defined in [13] is related to our slip coefficient by $\Lambda=2 \mu_{\mathrm{s}} \Xi$. From Fig. 1 , it is clear that the numerical swimming speed increases linearly with the slip coefficient. And our simulation results agree well with the analytical solution. Next, we study the effect of slip on the swimmer in

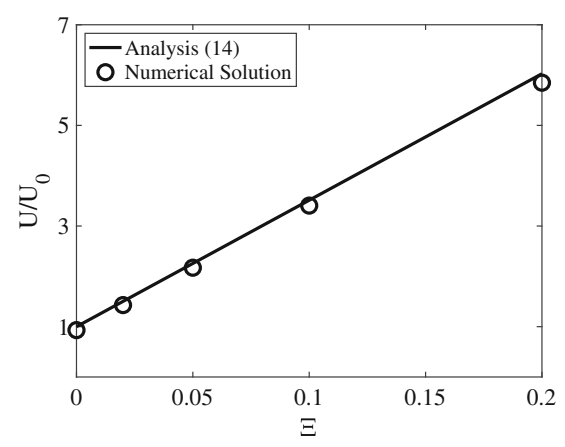

Fig. 1. Scaled swimming speed as a function of the slip coefficient: Taylor's sheet in a viscous fluid. 
a viscoelastic medium. We carry out simulations with different slip coefficients under three fixed values of the relaxation time $\lambda=2, \lambda=0.2$, and $\lambda=0.05$, respectively. The polymer viscosity is fixed at $\mu_{\mathrm{p}}=2$. The scaled swimming speed $\frac{\mathrm{U}}{\mathrm{U}_{0}}$ is plotted as the function of the slip coefficient in Fig. 2(a). Here the Deborah Number defined as De $=\lambda \omega$ is used to quantify the fluid elasticity. Note that in the plot, the analytical solution is plotted from (14), with $\mu_{\mathrm{s}}$ replaced by the total viscosity of the fluid $\mu_{\mathrm{s}}+\mu_{\mathrm{p}}$. The numerical results indicate that apparent slip always enhances the swimming speed in a viscoelastic fluid. It seems that for a fixed Deborah Number, the swimming speed increases linearly with the slip coefficient $\Xi$, which is similar to the swimmer in a viscous fluid. For the same slip coefficient, the swimming speed decreases with the increase of the fluid elasticity. As the Deborah Number De $\rightarrow 0$, the numerical solutions approach asymptotically to the analytical solution for the viscous fluid. Next, we fix the relaxation time $\lambda=0.2$ and study the influence of polymer viscosity on swimming under different slip coefficients. As shown in Fig. 2(b), when $\Xi=0$, the swimming speed decreases monotonically with the increase of $\mu_{\mathrm{p}}$. The result matches well with the analytical solution given by (15) [3]. When the slip coefficient is moderately increased to 0.02 , the swimming speed is not significantly impacted by the change of $\mu_{\mathrm{p}}$. And the variation is no longer monotone. For larger $\Xi$ values of 0.05 and 0.1 , greater values of $\mu_{\mathrm{p}}$ always lead to a faster swimmer, whose speed changes more dramatically with $\mu_{\mathrm{p}}$ than the one with smaller $\Xi$. Overall, the simulation results indicate that there exists a slip threshold beyond which the polymer viscosity can benefit swimming.

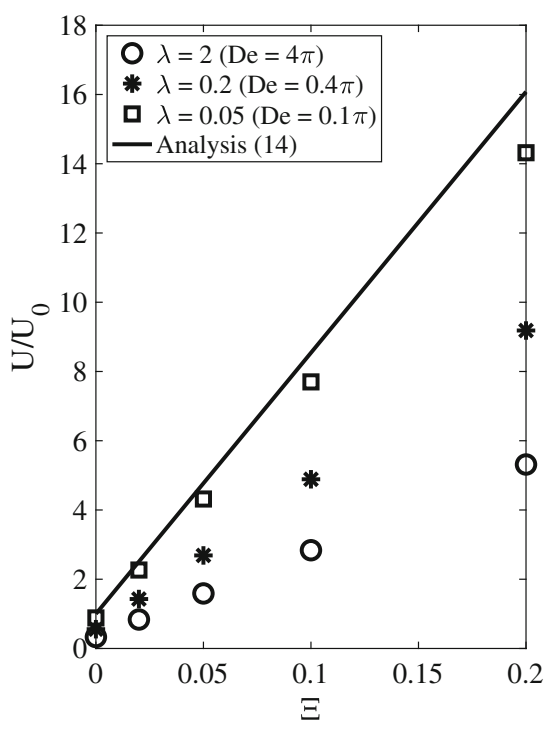

(a) $\mu_{\mathrm{p}}=2$

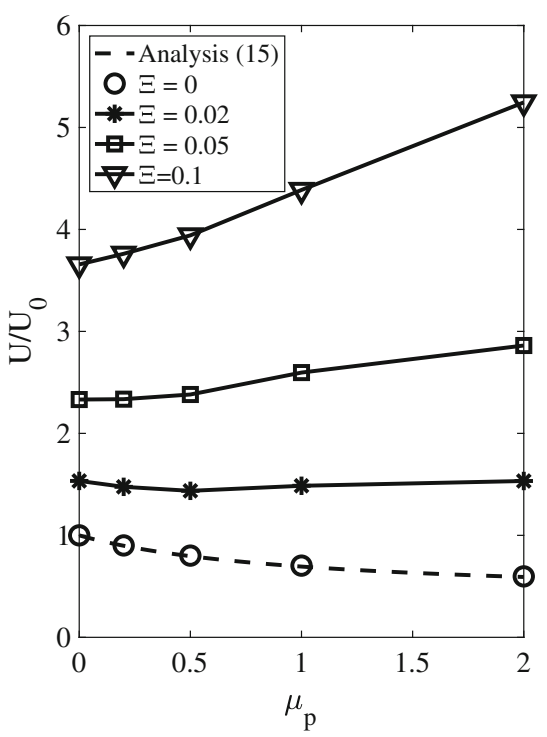

(b) $\lambda=0.2(\mathrm{De}=0.4 \pi)$

Fig. 2. Scaled swimming speed as a function of the slip coefficient (a) and polymer viscosity (b): Taylor's sheet in an Oldroyd-B fluid. 


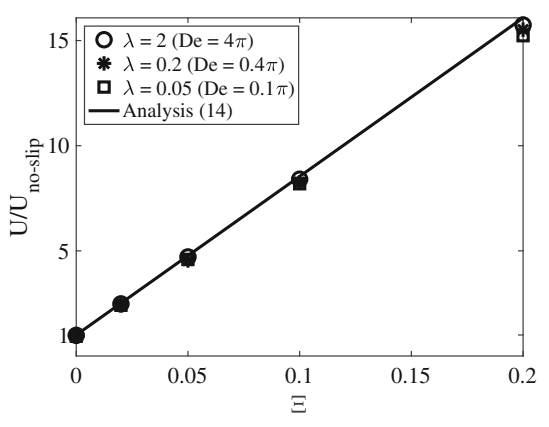

(a) $\mu_{\mathrm{p}}=2$

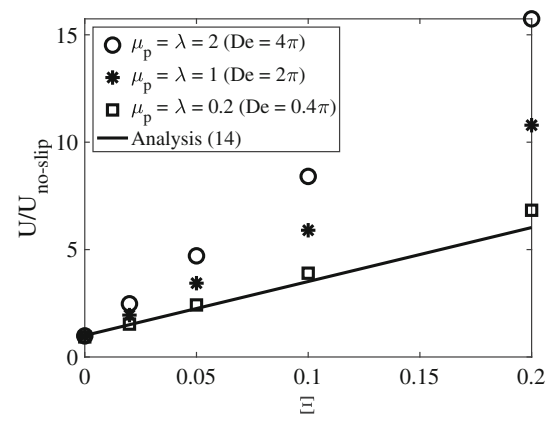

(b) $\frac{\mu_{\mathrm{p}}}{\lambda}=1$

Fig. 3. Relative boost in swimming speed as a function of the slip coefficient: Taylor's sheet in an Oldroyd-B fluid. Note that $\mathrm{U}_{\text {no-slip }}$ has different values for curves with different Deborah Numbers.

In Fig. 3(a), a different scaling is used for the same swimming speed U shown in Fig. 2(a). Here $\mathrm{U}_{\text {no-slip }}$ is the analytical second order swimming speed for an infinite sheet in an Oldroyd-B fluid (without slip) [3]:

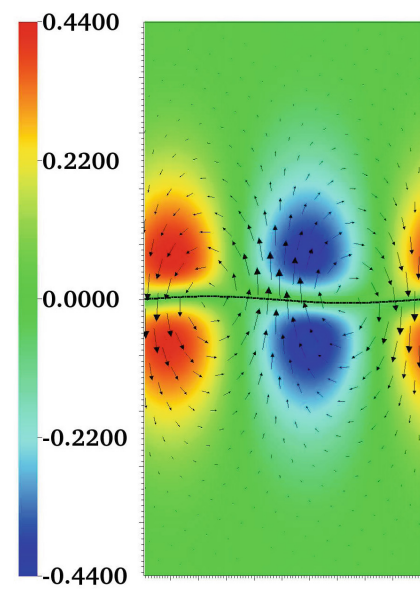

(a) $\Xi=0,\|\mathbf{u}\|_{\max }=0.075$

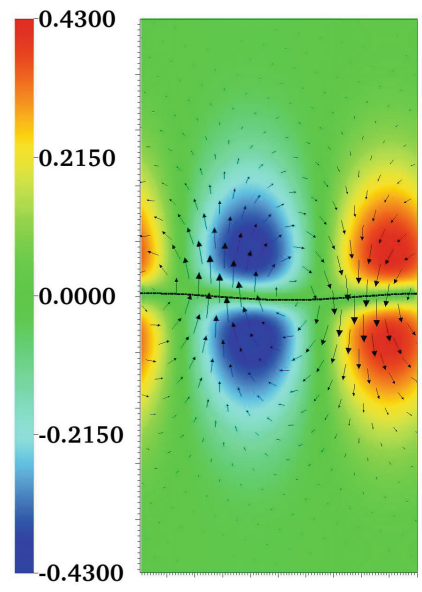

(b) $\Xi=0.2,\|\mathbf{u}\|_{\max }=0.073$

Fig. 4. Distribution of $\mathbf{u}$ and $\boldsymbol{\sigma}_{\mathrm{p}}^{12}$ at $\mathrm{t}=8$ for different $\Xi$. 


$$
\mathrm{U}_{\mathrm{no}-\mathrm{slip}}=\frac{1+\frac{\mu_{\mathrm{s}}}{\mu_{\mathrm{s}}+\mu_{\mathrm{p}}} \mathrm{De}^{2}}{1+\mathrm{De}^{2}} \mathrm{U}_{0}
$$

Therefore, $\frac{\mathrm{U}}{\mathrm{U}_{\text {no-slip }}}$ measures the relative slip boost for a swimmer in the same medium. Interestingly, for a fixed $\mu_{\mathrm{p}}$, the numerical results with different Deborah Numbers all match well with the analytical solution with $\mathrm{De}=0$. Therefore, for the range of parameters tested in this work, our results indicate that the relative slip boost for the infinite waving sheet is similar for a single phase viscous and a single phase viscoelastic fluid (with fixed fluid viscosity). In Fig. 3(b), the scaled speed is plotted for a fixed ratio of polymer viscosity to relaxation time $\frac{\mu_{\mathrm{p}}}{\lambda}=1$. Here the analytical solution is plotted from (14) without viscosity contribution from the polymer $\left(\mu_{\mathrm{p}}=0\right)$. For fixed $\mu_{\mathrm{s}}$ and $\omega$, the ratio $\frac{\mu_{\mathrm{p}}}{\lambda}$ measures the relative contribution of the polymeric stress to the force balance in fluid [5]. It is clear that for the same slip coefficient, the relative speed boost increases with the increase of Deborah Number. As the values of $\mu_{\mathrm{p}}$ and $\lambda$ decrease, the fluid behaves more like a viscous fluid with viscosity $\mu_{\mathrm{s}}$. In Fig. 4, the distributions of fluid velocity $\mathbf{u}$ and stress component $\boldsymbol{\sigma}_{\mathrm{p}}^{12}$ at $\mathrm{t}=8$ are plotted for two simulations both with $\mu_{\mathrm{p}}=2$ and $\lambda=0.2$. The one on the left has no-slip condition while the one on the right has slip coefficient $\Xi=0.2$. Compared with the no-slip case, the magnitude of $\boldsymbol{\sigma}_{\mathrm{p}}^{12}$ and $\mathbf{u}$ is slightly lower for the simulation with slip.

\section{Swimming in Viscoelastic Two-Fluid Mixture}

\subsection{Model Equations and Two-Phase IB Method}

In this section, we study the swimming sheet problem in a two-fluid mixture, which is modeled as a mixture of a viscous solvent phase (denote by s) and a viscoelastic network phase (denoted by $\mathrm{n}$ ). The viscous solvent fluid satisfies the standard no-slip condition on the swimmer while the viscoelastic network fluid can slip freely in the direction tangential to the swimmer. Two-fluid models of this kind have been widely used to describe dynamics of biofluids such as blood clot, biofilm and cytoplasm [10,23]. At any spatial location $\mathbf{x}$, the relative amounts of the two fluids are given by their volume fraction, $\theta_{\mathrm{s}}(\mathbf{x}, \mathrm{t})$ and $\theta_{\mathrm{n}}(\mathbf{x}, \mathrm{t})$ for the solvent and network, respectively. In this work, we treat $\theta_{\mathrm{s}}$ and $\theta_{\mathrm{n}}$ as model parameters with spatially uniform values constant in time. The solvent and network fluids move with their own velocity fields, $\mathbf{u}_{\mathrm{s}}(\mathbf{x}, \mathrm{t})$ and $\mathbf{u}_{\mathrm{n}}(\mathbf{x}, \mathrm{t})$. Mass conservation gives the incompressibility condition on the volume-averaged velocity:

$$
\nabla \cdot\left(\theta_{\mathrm{s}} \mathbf{u}_{\mathrm{s}}+\theta_{\mathrm{n}} \mathbf{u}_{\mathrm{n}}\right)=0 .
$$

For a small Reynolds number, the force balance equations for the two fluids are given by:

$$
\begin{gathered}
\nabla \cdot\left(\theta_{\mathrm{s}} \boldsymbol{\sigma}_{\mathrm{s}}\right)-\theta_{\mathrm{s}} \nabla \mathrm{p}+\xi \theta_{\mathrm{n}} \theta_{\mathrm{s}}\left(\mathbf{u}_{\mathrm{n}}-\mathbf{u}_{\mathrm{s}}\right)+\mathbf{f}_{\mathrm{s}}=0 \\
\nabla \cdot\left(\theta_{\mathrm{n}} \boldsymbol{\sigma}_{\mathrm{n}}\right)+\nabla \cdot\left(\theta_{\mathrm{n}} \boldsymbol{\sigma}_{\mathrm{p}}\right)-\theta_{\mathrm{n}} \nabla \mathrm{p}+\xi \theta_{\mathrm{n}} \theta_{\mathrm{s}}\left(\mathbf{u}_{\mathrm{s}}-\mathbf{u}_{\mathrm{n}}\right)+\mathbf{f}_{\mathrm{n}}=0 .
\end{gathered}
$$


Here, $\boldsymbol{\sigma}_{\mathrm{s}}$ and $\boldsymbol{\sigma}_{\mathrm{n}}$ are the viscous stress tensors for the solvent and network, respectively. $\boldsymbol{\sigma}_{\mathrm{p}}$ is the viscoelastic stress tensor for the network fluid. $\xi \theta_{\mathrm{n}} \theta_{\mathrm{s}}\left(\mathbf{u}_{\mathrm{n}}-\right.$ $\mathbf{u}_{\mathrm{s}}$ ) represents the frictional drag force between the two fluids due to relative motions where $\xi$ is the drag coefficient. $\mathbf{f}_{\mathrm{s}}$ and $\mathbf{f}_{\mathrm{n}}$ are force densities generated by immersed elastic structures on the two fluids. $\boldsymbol{\sigma}_{\mathrm{s}}$ and $\boldsymbol{\sigma}_{\mathrm{n}}$ are taken to be those of Newtonian fluids:

$$
\begin{aligned}
& \boldsymbol{\sigma}_{\mathrm{s}}=\mu_{\mathrm{s}}\left(\nabla \mathbf{u}_{\mathrm{s}}+\nabla \mathbf{u}_{\mathrm{s}}^{\mathrm{T}}\right)+\left(\lambda_{\mathrm{s}} \nabla \cdot \mathbf{u}_{\mathrm{s}}\right) \mathrm{I} \\
& \boldsymbol{\sigma}_{\mathrm{n}}=\mu_{\mathrm{n}}\left(\nabla \mathbf{u}_{\mathrm{n}}+\nabla \mathbf{u}_{\mathrm{n}}^{\mathrm{T}}\right)+\left(\lambda_{\mathrm{n}} \nabla \cdot \mathbf{u}_{\mathrm{n}}\right) \mathrm{I} .
\end{aligned}
$$

Here $\mathrm{I}$ is the identity tensor, $\mu_{\mathrm{s}}$ and $\mu_{\mathrm{n}}$ are the shear viscosities and $\lambda_{\mathrm{s}, \mathrm{n}}+2 \mu_{\mathrm{s}, \mathrm{n}} / d$ are the bulk viscosities of the solvent and network ( $d$ is the dimension). We choose $\lambda_{\mathrm{s}, \mathrm{n}}=-\mu_{\mathrm{s}, \mathrm{n}}$ so that the bulk viscosities in both phases are zero. The network fluid is treated as an Oldroyd-B fluid with constitutive equation given by (3), where $\mathbf{u}$ is replaced by network velocity $\mathbf{u}_{\mathrm{n}}$. In [14], an Immersed Boundary Method was proposed to simulate interactions between elastic structures and mixtures of two fluids. A penalty method was used to enforce the no-slip condition for both fluids on the elastic boundaries. In this work, we propose an extension to the method which allows the elastic structure to slip through one of the materials in the mixture. As shown in Fig. 5, the infinite sheet is represented by the immersed boundary $\Gamma^{\mathrm{s}}$, where the associated IB points $\mathbf{X}_{\mathrm{s}}(\mathrm{q}, \mathrm{t})$ move with local solvent velocity $\mathbf{u}_{\mathrm{s}}$ (no-slip condition). A "virtual IB" $\Gamma^{\mathrm{n}}$ is introduced to enforce the no-penetration condition for the network fluid on the boundary. Material points on the virtual IB are denoted by $\mathbf{X}_{n}(q, t)$, which move with local network velocity $\mathbf{u}_{\mathrm{n}}$. As indicated in the figure, each $\mathbf{X}_{\mathrm{n}}$ is connected by a stiff spring (with zero rest length) to a corresponding "anchor point" $\mathbf{X}_{\mathrm{n}}^{\mathrm{a}}$ located on $\Gamma^{\mathrm{s}}$. Similarly, each $\mathbf{X}_{\mathrm{s}}$ is connected to an anchor point $\mathbf{X}_{\mathrm{s}}^{\mathrm{a}}$ located on $\Gamma^{\mathrm{n}}$. The resulting force penalizes separation between $\Gamma^{\mathrm{s}}$ and $\Gamma^{\mathrm{n}}$ in the normal direction, without penalizing the relative motion tangential to the sheet surface. Using an analog of (7), Lagrangian force on $\Gamma^{\mathrm{s}}$ is distributed only to the solvent fluid and Lagrangian force on $\Gamma^{\mathrm{n}}$ is distributed only to the network fluid. In (17) and (18), the Eulerian force densities have the form $\mathbf{f}_{\mathrm{s}}=\theta_{\mathrm{s}} \mathrm{S} \mathbf{F}_{\mathrm{s}}^{\mathrm{o}}+\theta_{\mathrm{s}} \theta_{\mathrm{n}} \mathrm{S} \mathbf{F}_{\mathrm{s}}^{\mathrm{p}}$ and $\mathbf{f}_{\mathrm{n}}=\theta_{\mathrm{n}} \mathrm{S} \mathbf{F}_{\mathrm{n}}^{\mathrm{o}}+\theta_{\mathrm{s}} \theta_{\mathrm{n}} \mathrm{S} \mathbf{F}_{\mathrm{n}}^{\mathrm{p}}$. Here $\mathrm{S}$ is the force spreading operator defined before.

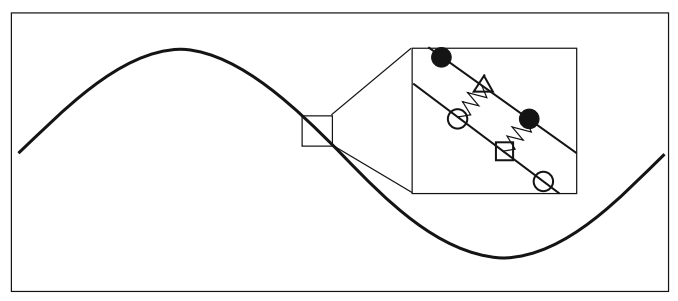

Fig. 5. Dual IB representation of an infinite swimmer $\bullet-\mathbf{X}_{\mathrm{s}}, \circ-\mathbf{X}_{\mathrm{n}}, \square-\mathbf{X}_{\mathrm{s}}^{\mathrm{a}}, \triangle-\mathbf{X}_{\mathrm{n}}^{\mathrm{a}}$. $\mathbf{X}_{\mathrm{n}}$ and $\mathbf{X}_{\mathrm{s}}$ are material points on the boundary. $\mathbf{X}_{\mathrm{s}}^{\mathrm{a}}$ and $\mathbf{X}_{\mathrm{n}}^{\mathrm{a}}$ are anchor points to enforce the no-penetration boundary condition for the network fluid. 
$\mathbf{F}_{\mathrm{s}}^{\mathrm{p}}$ and $\mathbf{F}_{\mathrm{n}}^{\mathrm{p}}$ are penalty forces on $\Gamma^{\mathrm{s}}$ and $\Gamma^{\mathrm{n}}$, respectively. At each IB point and the associated anchor point, we have $\mathbf{F}_{\mathrm{s}}^{\mathrm{p}}=-\mathbf{F}_{\mathrm{n}}^{\mathrm{p}}$. The spread contributions from the penalty forces are scaled by the product of the volume-fractions $\theta_{\mathrm{s}} \theta_{\mathrm{n}}$ so that no penalty force is applied if either of the volume fractions goes to zero. This also ensures that the total net penalty forces applied to the two fluids approximately add up to zero, provided that an IB point and its anchor point are always close (small normal separation between $\Gamma^{\mathrm{n}}$ and $\Gamma^{\mathrm{s}}$ ). Other Lagrangian forces $\mathbf{F}_{\mathrm{s}}^{\mathrm{o}}$ and $\mathbf{F}_{\mathrm{n}}^{\mathrm{o}}$ are scaled by the fluid's volume fraction after they are spread to that fluid. These include forces from the springs connecting an IB point to its two neighbors. Additionally, $\mathbf{F}_{\mathrm{s}}^{\mathrm{o}}$ also include forces from the springs connecting $\Gamma^{\mathrm{s}}$ to tether points with prescribed waving motion.

\section{$3.2 \quad$ Numerical Solutions}

To solve the model equations presented in the previous section, we use the same space-time discretization as described in Sect. 2.3. The time iteration scheme is given by:

1. From the boundary configurations $\mathbf{X}_{\mathrm{s}}\left(\mathrm{q}, \mathrm{t}^{\mathrm{k}}\right)$ and $\mathbf{X}_{\mathrm{n}}\left(\mathrm{q}, \mathrm{t}^{\mathrm{k}}\right)$, identify the anchor points $\mathbf{X}_{\mathrm{s}}^{\mathrm{a}}$ and $\mathbf{X}_{\mathrm{n}}^{\mathrm{a}}$ for all IB points on the two boundaries. Compute boundary forces $\mathbf{F}_{\mathrm{s}}^{\mathrm{o}}, \mathbf{F}_{\mathrm{n}}^{\mathrm{o}}, \mathbf{F}_{\mathrm{s}}^{\mathrm{p}}$, and $\mathbf{F}_{\mathrm{n}}^{\mathrm{p}}$ at $\mathrm{t}^{\mathrm{k}}$. Use the values to calculate the Eulerian force densities $\mathbf{f}_{\mathrm{s}}$ and $\mathbf{f}_{\mathrm{n}}$ on the two fluids.

2. Update the viscoelastic stress tensor $\boldsymbol{\sigma}_{\mathrm{p}}^{\mathrm{k}+1}$ using extrapolated network velocity at time level $\mathrm{t}^{\mathrm{k}+1 / 2}$.

3. Solve discrete versions of (16), (17) and (18) to get the values of $\mathbf{u}_{\mathbf{s}}, \mathbf{u}_{\mathrm{n}}$ and $\mathrm{p}$ at $\mathrm{t}^{\mathrm{k}+1}$.

4. Update the positions of all IB points by $\mathbf{X}_{\mathrm{j}}\left(\mathrm{q}, \mathrm{t}^{\mathrm{k}+1}\right)=\mathbf{X}_{\mathbf{j}}\left(\mathrm{q}, \mathrm{t}^{\mathrm{k}}\right)+\Delta \mathrm{t}\left(S_{\mathrm{h}}^{*}\right)^{\mathrm{k}} \mathbf{u}_{\mathrm{j}}^{\mathrm{k}+1}$ for $\mathrm{j}=\mathrm{s}, \mathrm{n}$.

In step 1, $\mathbf{F}_{\mathrm{s}}^{\mathrm{p}}$ and $\mathbf{F}_{\mathrm{n}}^{\mathrm{p}}$ are computed at and spread from all IB and anchor points. For a specific $\mathbf{X}_{\mathrm{s}}$, the associated anchor point $\mathbf{X}_{\mathrm{s}}^{\mathrm{a}}$ is defined as the point on the piece-wise linear boundary $\Gamma^{\mathrm{n}}$ such that $\left\|\mathbf{X}_{\mathrm{s}}-\mathbf{X}_{\mathrm{s}}^{\mathrm{a}}\right\|$ is the shortest distance between $\mathbf{X}_{\mathrm{s}}$ and $\Gamma^{\mathrm{n}}$. The anchor point $\mathbf{X}_{\mathrm{n}}^{\mathrm{a}}$ on $\Gamma^{\mathrm{s}}$ for $\mathbf{X}_{\mathrm{n}}$ is identified similarly. In step 3, a multigrid preconditioned GMRES solver is used to solve the linear system [22]. All simulation parameters such as computational domain, grid size and time step are the same as ones used in Sect. 2.3. For all simulations, we set the viscosity values to $\mu_{\mathrm{s}}=\mu_{\mathrm{n}}=1.0$. In the first set of test, we fix the drag coefficient $\xi=1.0$ and fluid volume fractions $\theta_{\mathrm{s}}=\theta_{\mathrm{n}}=0.5$. The influence of relaxation time on swimming is studied for three different values of polymer viscosities $\mu_{\mathrm{p}}=0.5$, $\mu_{\mathrm{p}}=2$ and $\mu_{\mathrm{p}}=4$. In Fig. $6(\mathrm{a})$ and (b), the relative velocity $\mathbf{u}_{\mathrm{n}}-\mathbf{u}_{\mathrm{s}}$ and the stress component $\boldsymbol{\sigma}_{\mathrm{p}}^{12}$ are plotted for $\mu_{\mathrm{p}}=0.5$ and $\mu_{\mathrm{p}}=2$, respectively, at $\mathrm{t}=12$. In both plots, the relative velocity is approximately tangent to the sheet, indicating the boundary condition is properly enforced. With a larger polymer viscosity, the stress component has larger magnitude while the motion of the network relative to the solvent fluid is less significant. The scaled swimming speed is shown in Fig. 7(a) as the function of $\lambda$ for different values of $\mu_{\mathrm{p}}$. The 


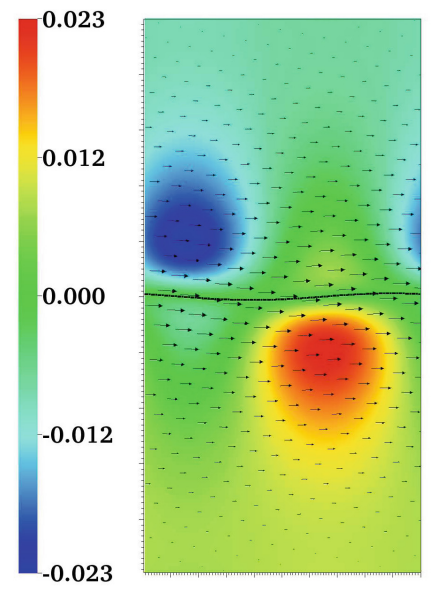

(a) $\mu_{\mathrm{p}}=0.5,\left\|\mathbf{u}_{\mathrm{n}}-\mathbf{u}_{\mathrm{s}}\right\|_{\max }=0.05$

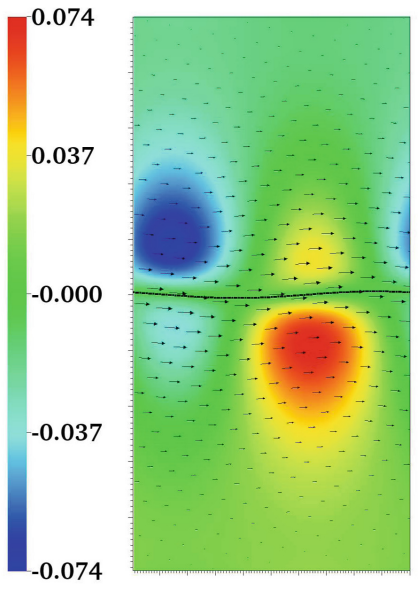

(b) $\mu_{\mathrm{p}}=2,\left\|\mathbf{u}_{\mathrm{n}}-\mathbf{u}_{\mathrm{s}}\right\|_{\max }=0.043$

Fig. 6. Distribution of $\mathbf{u}_{\mathrm{n}}-\mathbf{u}_{\mathrm{s}}$ and $\boldsymbol{\sigma}_{\mathrm{p}}^{12}$ for $\lambda=2$ at $\mathrm{t}=12$.

plots indicate that the sheet always swims much faster in the mixture than in a viscous fluid, even when the mixture contains a highly elastic network. For fixed polymer viscosity, the increase of the network elasticity monotonically hinders the swimming speed. For a fixed $\lambda$, the sheet moves faster in mixtures with larger values of $\mu_{\mathrm{p}}$. The speed enhancement due to polymer viscosity is more significant for less elastic mixture. Next, we carry out simulations in which both $\mu_{\mathrm{p}}$ and $\lambda$ are varied while the values of their ratio $\frac{\mu_{\mathrm{p}}}{\lambda}$ remain fixed. As seen from Fig. $7(\mathrm{~b})$, with fixed $\frac{\mu_{\mathrm{p}}}{\lambda}$, the swimming speed is always moderately enhanced when $\mu_{\mathrm{p}}$ and $\lambda$ increase with the same rate. Together with the data shown in Fig. 2(b), our simulation results suggest that for a swimmer that is allowed to slip through a viscoelastic material (or mixture of materials), the speed of locomotion is dependent in a nontrivial way on both the viscosity and elasticity of the material. In Fig. 8(a), the swimming speed is plotted as the function of the drag coefficient $\xi$ for $\theta_{\mathrm{n}}=0.5$. The sheet moves slower with the increase of drag. For a drag coefficient of $\xi=10^{4}$, the swimming speed is about $60 \%$ of that in a viscous fluid. In Fig. 8(b), $\frac{\mathrm{U}}{\mathrm{U}_{0}}$ is plotted for different network volume fraction $\theta_{\mathrm{n}}$ with $\xi$ fixed at 1 . The increase of the network volume fraction in the mixture leads to significant swimming speed-ups. In a separate test with no-slip network, we observe smaller swimming speed with the increase of $\theta_{\mathrm{n}}$ (result not shown). 


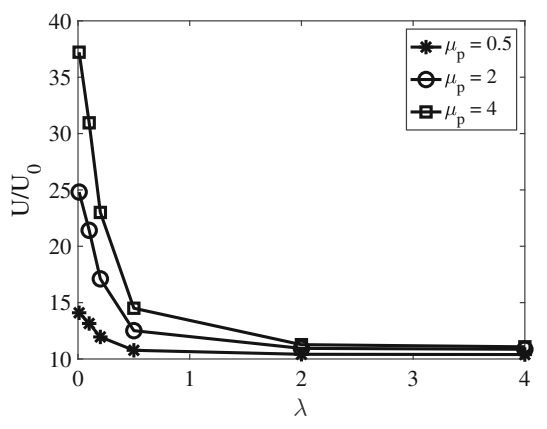

(a) $\mu_{\mathrm{p}}=0.5,2$ and 4

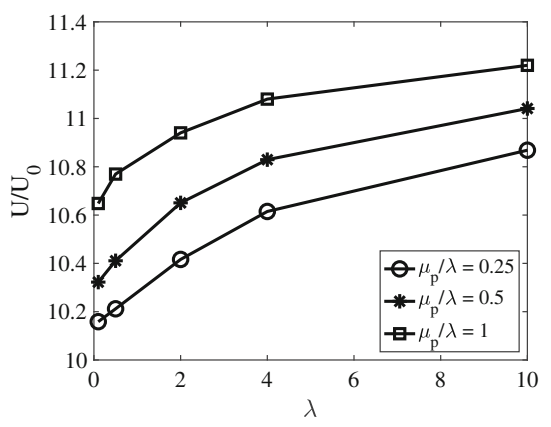

(b) $\frac{\mu_{\mathrm{p}}}{\lambda}=0.25,0.5$, and 1

Fig. 7. Scaled swimming speed as a function of relaxation time $\lambda$ : Taylor's sheet in a two-fluid mixture with no-slip solvent fluid and free-slip network fluid.

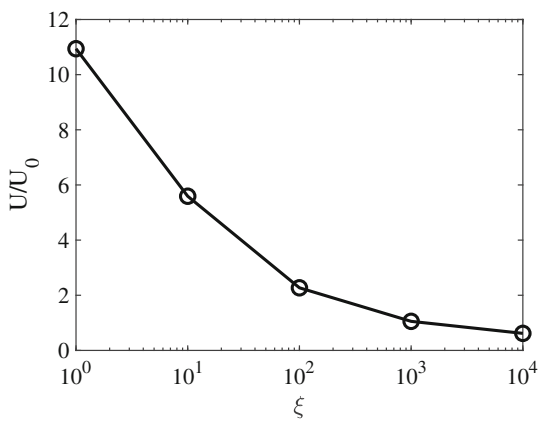

(a) $\theta_{\mathrm{n}}=0.5$

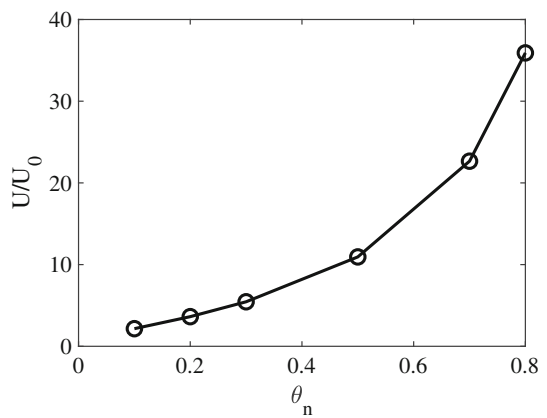

(b) $\xi=1$

Fig. 8. Scaled swimming speed as a function of the drag coefficient (a) and the network volume fraction (b): Taylor's sheet in a two-fluid mixture. $\mu_{\mathrm{p}}=2, \lambda=2$.

\section{Conclusion}

We simulate the infinite swimming sheet problem in complex fluids under slip boundary conditions with extensions of the classical IB method. For swimmers in a viscoelastic fluid, interpolated fluid velocities are modified using tangential components of the Lagrange force to account for the partial slip condition. This can be thought as the single-phase version of the force calculation strategy proposed in [15]. In a viscoelastic two-fluid mixture, a dual IB representation of the immersed structure is used where the free-slip condition is enforced through a penalty method. Instead of the projection-based fractional step methods as used in [15], we solve the momentum equations and the incompressibility constraint simultaneously. This makes it more straightforward to enforce the velocity boundary conditions. Furthermore, our method can be directly applied to problems where fluid volume fractions are spatially variable. For such problems, 
methods for Stokes equations that decouple the velocity and the pressure, such as the pressure-Poisson formulation, can not be used. Our numerical results show that: (1) Slip may lead to substantial speed enhancement for the swimmer in a viscoelastic fluid or two-fluid mixture relative to the swimmer in a no-slip viscous fluid. (2) For a viscoelastic fluid with fixed viscosity and relaxation time, the swimming speed increases linearly with the slip coefficient. With fixed viscosity and slip coefficient, the swimming speed decreases with the increase of relaxation time (fluid elasticity). (3) While polymer viscosity always hinders swimming for a no-slip viscoelastic fluid, it can benefit the swimmer in a viscoelastic fluid if the slip coefficient is large enough. (4) In a two-fluid mixture where the swimmer is allowed to slip freely through the viscoelastic network, speed enhancement can be obtained by reducing the drag coefficient, increasing the polymer viscosity, and increasing the network volume fraction.

\section{References}

1. Berg, H.: E. Coli in Motion. Springer, New York (2004). https://doi.org/10.1007/ b97370

2. Fauci, L., Dillon, R.: Biofluidmechanics of reproduction. Ann. Rev. Fluid Mech. 38, 371-394 (2006)

3. Lauga, E.: Propulsion in a viscoelastic fluid. Phys. Fluids 19(8), 083104 (2007)

4. Riley, E., Lauga, E.: Small-amplitude swimmers can self-propel faster in viscoelastic fluids. J. Theor. Biol. 382, 345-355 (2015)

5. Teran, J., Fauci, L., Shelley, M.: Viscoelastic fluid response can increase the speed and efficiency of a free swimmer. Phys. Rev. Lett. 104(3), 038101 (2010)

6. Shen, X., Arratia, P.: Undulatory Swimming in viscoelastic fluids. Phys. Rev. Lett. 106, 208101 (2011)

7. Espinosa-Garcia, J., Lauga, E., Zenit, R.: Fluid elasticity increases the locomotion of flexible swimmers. Phys. Fluids 25, 031701 (2013)

8. Thomases, B., Guy, R.: Mechanisms of elastic enhancement and hindrance for finite length undulatory swimmers in viscoelastic fluids. Phys. Rev. Lett. 113(9), 098102 (2014)

9. Thomases, B., Guy, R.: The role of body flexibility in stroke enhancements for finite-length undulatory swimmers in viscoelastic fluids. J. Fluid Mech. 825, 109132 (2017)

10. Cogan, N., Guy, R.: Multiphase flow models of biogels from crawling cells to bacterial biofilms. HFSP J. 4(1), 11-25 (2010)

11. Barnes, H.: A Review of the slip (Wall Depletion) of polymer solutions, emulsions and particle suspensions in viscometers. J. Non-Newton. Fluid Mech. 56(3), 221251 (1995)

12. Fu, H., Shenoy, V., Powers, T.: Low-Reynolds-number swimming in gels. Europhys. Lett. 91(2), 24002 (2010)

13. Man, Y., Lauga, E.: Phase-separation models for swimming enhancement in complex fluids. Phys. Rev. E 92, 023004 (2015)

14. Du, J., Guy, R., Fogelson, A.: An immersed boundary method for two-fluid mixtures. J. Comput. Phys. 262, 231-243 (2014)

15. Lee, P., Wolgemuth, C.: An immersed boundary method for two-phase fluids and gels and the swimming of C. elegans through viscoelastic fluids. Phys. Fluids 28(1), 011901 (2016) 
16. Peskin, C.: The immersed boundary method. Acta Numerica 11, 479-517 (2002)

17. Sochi, T.: Slip at fluid-solid interface. Polym. Rev. 51(4), 309-340 (2011)

18. Taylor, G.: Analysis of the swimming of microscopic organisms. Proc. Roy. Soc. A 209, 447-461 (1951)

19. Lauga, E., Powers, T.: The hydrodynamics of swimming microorganisms. Rep. Prog. Phys. 72(19), 096601 (2009)

20. Williams, H., Fauci, L., Gaver III, D.: Evaluation of interfacial fluid dynamical stresses using the immersed boundary method. Disc. Continuous Dyn. Syst. Ser. B 11(2), 519-540 (2009)

21. Vanka, S.: Block-implicit multigrid solution of Navier-Stokes equations in primitive variables. J. Computat. Phys. 65(1), 138-158 (1986)

22. Wright, G., Guy, R., Du, J., Fogelson, A.: A high-resolution finite-difference method for simulating two-fluid, viscoelastic gel dynamics. J. Non-Newton. Fluid Mech. 166, 1137-1157 (2011)

23. Du, J., Fogelson, A.: A two-phase mixture model of platelet aggregation. Math. Med. Biol. 35(2), 225-256 (2018) 